\title{
Source Localization Using A Non-Traditional Three-Dimensional Ocean Modeler
}

\author{
C.M. DeAngelis \\ Naval Undersea Warfare Center Division \\ Code 2222, Building 1171-3 \\ Newport, Rhode Island, 02841 \\ deangelis@ada.npt.nuwc.navy.mil
}

\author{
R.W. Green \\ Computer and Information Science Department \\ University of Massachusetts Dartmouth \\ North Dartmouth, Massachusetts 02747 \\ green@cis.umassd.edu
}

\begin{abstract}
A real-time source localization system comprised of traditional signal processing and nontraditional artificial neural network technologies is presented. A technique for multiple receiver data fusion is also discussed along with issues regarding solution sensitivity and three dimensional contact state parameter estimation. This synergistic coupling of technologies provides scientists with a new tool for solving real-time problems.
\end{abstract}

\section{INTRODUCTION}

Source localization in the ocean environment is anything but trivial. Space, power, and weight restrictions alone make many functional systems unsuited for anything other than laboratory installations. To compound matters further, the computational requirements of complex, dynamic ocean models prohibit any kind of real-time response from systems that are suited for vessel installment. Therefore, a different approach to source localization needs to be developed.

The scientific community has demonstrated through the synergistic coupling of traditional signal processing techniques with non-traditional information processing algorithms that these hurdles can be overcome, and real-time system performance is achievable even when only a limited amount of computer resources are available. One of the popular non-traditional algorithms widely used is the artificial neural network; it offers speed, robustness, adaptability, and the ability to approximate system functions that are not well definable or are even unknown [1].

The following sections present such a synergistic coupling of technologies in modeling a threedimensional ocean. In doing so, a viable method for source localization in real-time is demonstrated. Traditional signal processing techniques are used to estimate a time series of angular directions to a source for one or more receivers. This information is then processed by an artificial neural network to provide an estimate of source location as well as velocity in three dimensions. These estimates are continuously updated and refined as more information becomes available. Knowledge about source, medium, and receiver characteristics and kinematics is also exploited in the model.

In addition to speed of execution, the artificial neural network provides a robust implementation of multiple receiver signal fusion. Measurements from all receivers are analyzed to determine not only a more accurate angular direction to the source, but more importantly a bounding region on where the source may reside. Once this region is estimated, it is focused on and validated. Thus, the artificial neural network dynamically adapts its attention to areas that have a higher probability of source containment. It does not however, totally ignore any area corresponding to a minimum containment probability since these regions may become more important as time goes on.

This new approach to problem solving demonstrates the use of non-traditional processing algorithms to provide modules of a system that are either too computationally intensive to be performed by traditional techniques, or not well represented or approximated by mathematics.

\section{SOURCE LOCALIZATION}

\section{Background}

Source localization in the underwater environment is at best difficult. To begin with, ocean characteristics such as the Sound Velocity Profile (SVP), ocean floor reflection and absorption properties, along with various scattering effects, need to be accurately represented. In addition, potential propagation paths of the received signals must be determined, otherwise all estimates of location and motion will likely be erroneous. There still remains the issues involving the extraction of measurements from received signals, correlation of these measurements to a single or multiple contacts, and the fusion of data from multiple receivers.

A number of models have been developed and refined for the accurate approximation of sound propagation in the ocean environment [2]. The 
scientific community has also offered a number of signal processing algorithms tailored for beam steering and direction finding [3]. Collectively, these techniques provide a potentially highly accurate time series of measurements containing angular directions to a contact. The problems arise in fusing and correlating these measurements to provide accurate source localization.

Many traditional processing techniques are highly computationally intensive, requiring a tremendous amount of processing horsepower to provide an inkling of real-time response. With the recent advancements in the state-of-the-art central processing units, this is becoming less and less of an obstacle. Unfortunately, the problem of algorithm initialization is not as easily rectified. Many of the source localization algorithms need to be initialized close to the solution being sought in order to iterate to a stable convergence. To compound matters further, the confidence in this initialization is often indeterminable. Furthermore, a number of these algorithms only provide a quantitative result; thus, no feel for the sensitivity of the solution to small perturbations is available. It is clear that a new technique to source localization is needed.

\section{The Speed-Strip Method}

Assuming that methods exist for the extraction of a time series of angular measurements to a source from one or more receivers, there still exists the formidable task of correlating this information and providing an accurate estimate of source position and velocity. One simple and widely accepted method for correlating these measurements is known in the Naval community as "speed-strip-fitting". This technique provides an estimate of contact location and velocity by imposing a "constant-motion" constraint. Simply put, if a contact maintains a constant heading and a constant speed, then its straight-line trajectory will traverse equal distances in equal time intervals, as shown in figure-1a. Thus, a consistent spacing between the angular measurements is sought, depicted by vector " $A$ " in figure- $1 \mathrm{~b}$.

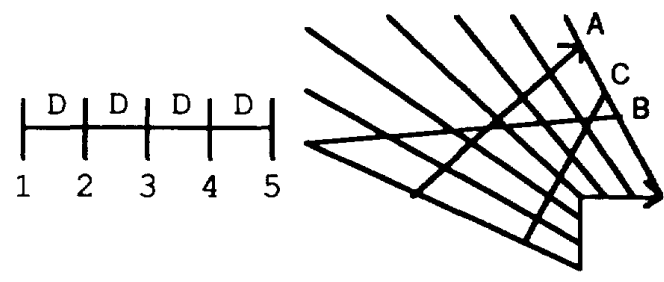

(a)

(b)

Figure-1: a) Equal distances being traversed in equal time intervals, b) Speed-strip match for vector "A", and mismatch for vectors " $B$ " and " $C$ ".
In operation, this multiple hypothesis testing technique uses a number of strips of paper, each with a set of equally spaced marks corresponding to a respective contact speed, to physically search for a consistent spacing between the angular measurements. Since each strip corresponds to a different speed, the name "speed-strip-fitting" was coined for this technique.

\section{Multiple Receiver Information Fusion}

Little effort needs to be spent in showing that exploiting information from multiple receivers is better than separately processing information from an individual receiver. The formidable task is to fuse the angular measurements provided by each receiver in the best possible way. It can shown that the simple process of overlaying the projections of the angular measurements from multiple receivers onto a reference surface, inherently provides the foundation for data fusion.

Figure-2 depicts the process of fusing angular measurements from two receivers. It is as simple as superimposing the projected vectors corresponding to similar time intervals. This can be done for each receiver; however, care must be taken in properly aligning the images to the reference surface.

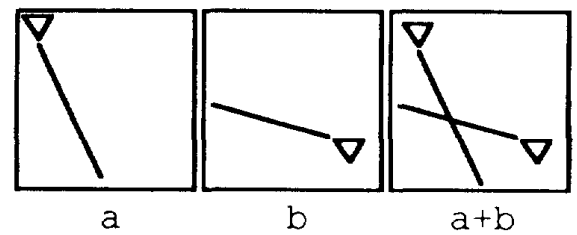

Figure-2: Fusion of angular measurements from two receivers.

The degree of fusion may range from soft to hard as shown in figure-3. Soft-fusion would restrict only one of the angular projections for a given time interval to line up with a mark on a speed-strip (Figure-3a). Hard-fusion, on the other hand, would require all angular projections for a given time interval to cross at a speed-strip mark (Figure-3b). Thus, soft-fusion often results in producing more possible speed-strip fits than hard-fusion. Other variations are also possible; therefore, the degree of fusion is variable according to the confidence in, or the availability of, the received data. Furthermore, with the right configuration of receivers, it is often possible to estimate all of the location and motion parameters of interest from a minimum number of measurements (Figure-3b) [4]. 


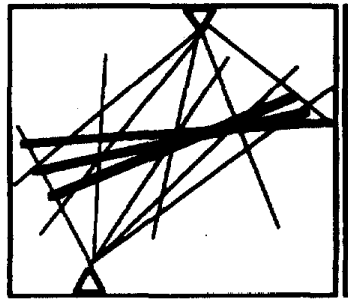

(a)

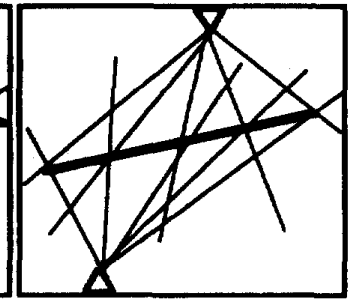

(b)
Figure-3: a) Soft fusion, b) Hard fusion.

\section{A Three-Dimensional Extension}

Speed-strip-fitting is typically done in two dimensions since the operator only has a twodimensional surface to wort on. This does not mean, however, that the model cannot be extended to three dimensions. Instead of selecting a depth and constraining the search to be in the remaining two dimensions, the search is done in all three dimensions; thus giving rise to a type of 3-D tic-tactoe game, where the vertical axis is now a parameter. This requires the angular measurements to be projected into an imaginary volume, instead of onto a surface, prior to fitting the speed strips. Today's laser and holographic technologies would easily accommodate such a conceptual system.

It is apparent that this extension to the third dimension could easily tax the efficiency of even the best speed-strip-fitters. What is needed is an automated approach to speed-strip-fitting. Since this task involves the human vision system, it only seems natural that we examine the vision system for inspiration in developing an automated speed-stripfitter.

\section{VISION}

The human vision system has been genetically constructed to determine such features as color, motion, and edges. If we could only see equally spaced points in a volume, the task of speed-stripfitting would be as simple as determining black from white. Although our vision system lacks proficiency in this area, it does offer a model for the development of such a system.

By oversimplifying the vision system, we see that it embodies three levels of processing: the receiving of a signal and retinal stimulations, the correlation of these stimulations, and the association of meaning to the correlation (Figure-4a). As with our automated speed-strip-fitter, it can be constructed in three levels: the receiving of a signal and projection of angular measurements, the correlation of these measurements, and the association of meaning to the correlation
(Figure-4b). In the latter, the correlation provides the detection of vectors containing equally spaced points and the meaning associated is a respective contact state (range, course, speed, and the like). Thus, in both cases there are three distinct levels. In the text that follow, these levels will be called: stimulation, correlation, and estimation.

Our source localization model is a modification of the human vision system model; all that needs to be done is a rewiring of the neuron connections to provide the detection of features such as range, course, and speed instead of color, motion, and edges. This rewiring embodies the imposed "constant-motion" constraint described above. This Neurally Inspired Contact Estimation (NICE) System is discussed in the following section.

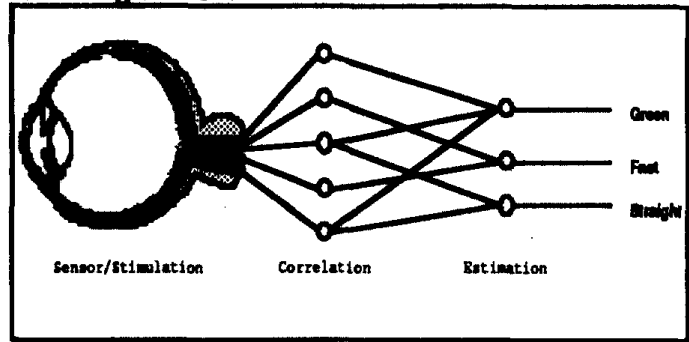

(a)

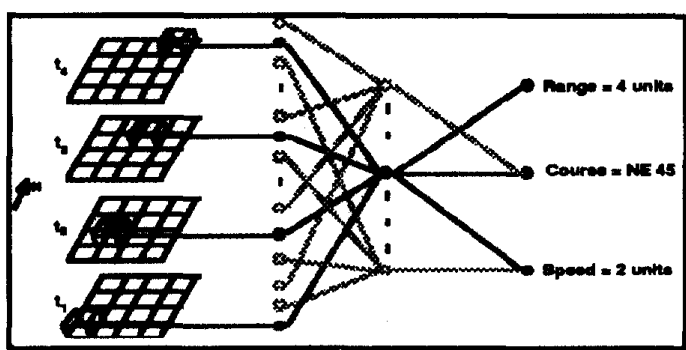

(b)

Figure-4: a) Human vision system, b) Speed-strip "vision" system.

\section{VISION AND SOURCE LOCALIZATION}

\section{A NICE Model}

The NICE model of source localization comprises three levels of simple processing units. These simple processing units are similar to those in the brain in that they form a weighted sum of the information at hand and respond according to some predetermined often non-linear function of that sum. A simplified version of the model's three levels is presented next. Issues regarding three-dimensional fusion are also discussed. A more detailed description of the architecture of NICE can be found in $[5,6]$. 


\section{Three NICE Levels}

The first level of the NICE model takes a time series of angular measurements and projects them onto a grid of neurons, thus stimulating the system. In three dimensions, this would be a volume of neurons, a three-dimensional retina. There is a retinal image corresponding to each measurement from each receiver. Fusion may be provided by boolean ANDing, ORing, or forming a weighted average of all images corresponding to related time intervals. This greatly compresses the amount of information into a handful of retinal images.

After these retinal images are formed, the second level of processing units looks for equally spaced points across the images. Theoretically, there would be a single neuron for each possible line vector (length and orientation) that may be in an image. This is a finite number since the images are stored in matrix form, thus having a specified number of columns and rows. In three dimensions, the length and orientation of the possible line vectors would need to span a volume. Note that there are restrictions on contact speeds; therefore dramatically reducing the exhaustive number of combinations to a realistic and manageable amount. Exploiting other information relating to source, media, or receiver characteristics would reduce this further.

In its simplest form, the neurons in the correlation level are boolean AND gates. In a more robust implementation, they would respond proportionally to the degree of compliance for a respective contact traversal across the images. In the case of the boolean AND, all images must provide evidence of equally spaced points; in a weighted case it may be: five out of six, or eight out of ten images giving evidence to the existence of a line vector. The output of the correlation layer is a vector of neuron intensity levels (1's and 0 's in the boolean AND case) corresponding to the existence of valid line vectors.

Contact state may now be estimated from the correlation vector. Since each correlation neuron corresponds to a specific course and speed pair, and multiple neurons representing similar line vectors are active, source position and motion must be estimated. If only a single correlation neuron were active, then no averaging would be required. A simple, yet effective, technique is to average all of the speeds (Figure-5a). This may also be done for the estimation of bearing. One must be careful when averaging since the distributions may be multi-modal; that is, there may be two or more distinct groups of speeds that should be independently averaged [7]. If collectively averaged, the results may be a value that did not exist in the initial distribution, as shown in figure-5b.

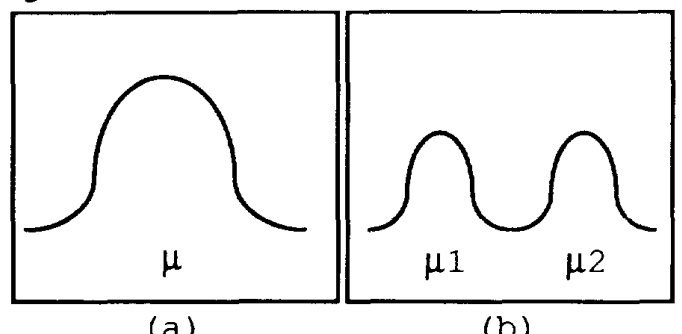

(a)

(b)

Figure-5: a) Uni-modal distribution, b) Bi-modal distribution.

\section{NICE Operation}

The NICE estimates are quantitative, but a qualitative view is also provided by looking at the distribution of the state parameters. This view gives tremendous insight into the sensitivity and confidence of the estimates. In addition, the standard deviations of the estimates may be computed and provided. This in itself gives a measure of parameter sensitivity. Furthermore, as more data is received, the estimates are updated and the rate of standard deviation decay can be monitored.

Since this model is made up of simple processing units (boolean in its simplest form), execution is very fast. Furthermore, the inherent parallelness makes it a prime candidate for the state-of-the-art distributed array processors; easily providing real-time performance. In addition, NICE offers a solution to the initialization problem on many traditional algorithms. It was not developed to replace traditional techniques, but rather to work with them. Overall, the NICE system offers a viable and robust method for real-time source localization.

\section{SUMMARY AND CONCLUSIONS}

NICE represents a new approach to problem solving. Specifically, it demonstrates the use of nontraditional algorithms, like artificial neural networks, to provide parts of a system that are either too computationally intensive to be performed by traditional techniques, or not well represented or approximated by mathematics. It incorporates algorithms across technologies in solving the source localization problem, and provides researchers with some insight into non-traditional processing capabilities.

No one technology will solve all our problems; it will only be through the continued exploitation of these synergistical systems that we can even hope to solve the problems of today and tomorrow. 


\section{ACKNOWLEDGMENT}

The authors would like to thank there supervisor, William Navin who, since 1988, has provided continuous encouragement, support, and advice on exploring neural networks and their application.

\section{REFERENCES}

[1] Rumelhart D. E. and J. L. McClelland, Parallel Distribused Processing, MIT Press, Cambridge, Massachusetts, 1988.

[2] Kinsler L. E. et. al., Fundamentals of Acoustics, John Wiley \& Sons, New York, 1982.

[3] Shanmugan K. S. and A. M. Breipohl, Random Signals: Detection, Estimation and Data Analysis, John Wiley \& Sons, New York, 1988.

[4] Nardone S. C. and V. J. Aidala, "Observability Criteria For Bearings-Only Target Motion Analysis", IEEE Transactions on Aerospace and Electronic Systems, Vol. AES-17, No. 2, March 1981.

[5] DeAngelis C. M. and R. W. Green, "An Architected Neural Network For Contact State Estimation", IEEE Proceedings of OCEANS 92, vol. 1, pp. 153-157.

[6] C. M. DeAngelis and R. W. Green, "Constructing Neural Networks for Contact Tracking", Neural Networks for Signal Processing - Proceedings of the 1992 IEEE Workshop.

[7] K. F. Gong, S. E. Hammel, S. C. Nardone and A. G. Lindgren, "Three dimensional Contact Parameter Estimation", Proceedings of the Sixteenth Asiloma Conference on Circuits, Systems and Computers, November 1982. 